\title{
Measurement of a micro-scale fluid physical properties using torsional vibration of a micro shaft
}

\author{
Mina Ghanbari ${ }^{*}$, Siamak Hossainpour ${ }^{2}$, Ghader Rezazadeh ${ }^{3}$ \\ ${ }^{1}$ Mechanical Engineering Department, Engineering Faculty of Khoy, Urmia University, Urmia, Iran \\ ${ }^{2}$ Mechanical Engineering Department, Sahand University of Technology, Tabriz, Iran \\ ${ }^{3}$ Mechanical Engineering Department, Faculty of Engineering, Urmia University, Urmia, Iran
}

Corresponding Author Email: m.ghanbari@urmia.ac.ir

https://doi.org/10.18280/mmc_b.870407

Received: 14 July 2018

Accepted: 14 December 2018

\section{Keywords:}

MEMS, micropolar theory, micro-scale fluid, torsional vibration

\begin{abstract}
The purpose of this study is presenting a novel micro-electromechanical (MEM) sensor for measurement of a micro-scale fluid physical properties. A mathematical model is proposed for this study which consists of a micro-shaft with one end fixed and a sensing element in the form of a cylinder at its free end. The fluid is bounded between the micro-cylinder as sensing element and the outer fixed cylinder. As fluids behave differently in micro-scale than macro, the fluid in the gap is modeled based on micro-polar fluid theory. The sensor can be actuated torsionally via applying an AC voltage to the pair of capacitive plates situated around the micro-shaft and the outer fixed cylinder. After deriving the equations of motion of the micro-shaft and also micro-scale fluid media, these coupled partial differential equations have been solved simultaneously using Galerkin based reduced order model. The dynamic response of the micro-shaft for different exciting frequencies has been investigated. It has been shown that inertial and damping effects of fluid, causes resonance frequency and resonance amplitude of the shaft to decrease. By calculating resonance frequency and resonance amplitude changes, physical properties of a fluid can be measured. Effects of geometrical parameters of the sensing element on the force response of the sensor have also been studied. Through this study it was found that a sensor with large surface area of sensing element and small fluid gap, could measure fluid properties with high accuracy.
\end{abstract}

\section{INTRODUCTION}

The importance of fluids physical properties can be seen in many branches of industry as food and pharmaceutical industries. For example, knowledge of viscosity is an important property in fluid dynamics because it is a key factor in determining the amount of fluid that can be transported in a pipeline during a specific period of time. Also, liquid viscosity in chemical process design makes it one of the most measured transport properties. Various classical methods have been presented for measurement of fluids physical properties. Magneto restrictive wave guide viscometers work based on the retardation of a torsion sound wave through a solid [1]. In vibrating cylindrical densitometers and viscometers, the motion of sensor is in relation with the fluid viscosity and density. These vibration-based sensors work on the force required to induce the sensor to move [1]. Other vibrationbased sensors are crystal resonator [2] and ultrasonic plate waves [3].

Recently, Micro-electro mechanical systems (MEMS) due to their advantages have become an interesting tpoic for study among researchers. Micro-scale devices have several advantages. They can be produced at low cost, in small size and with low-energy consumption and fast response time. So they can be used in diverse fields of engineering and science. micropumps, micromirrors, micro-accelerometers and microsensors are examples of micro-scale devices [4-7].

As solid-fluid interaction can be seen in many micro devices, many type of researches have been done on investigating the effect of fluid on the behavior of the micro structure. Ghanbari et.al studied squeeze film damping phenomenon in a microresonator by applying non-classical theories [8]. In another work, Ghanbari et al. investigated thin film damping in microbeam resonator based on micropolar theory [9]. Rezazadeh and Ghanbari studied the effect of fluid on the frequency response of the vibrating micro-beam and presented the proposed model as a MEM- based sensor for simultaneous measurement of fluids viscosity and density [10].

In most of micro devices as micro-sensors, microcantilevers are applied as sensing mechanism due to their advantages of having high sensitivity, quick response time and low power requirement [11-13]. Many researches have been done on using micro-cantilevers in sensing devices especially in measurement sensors for determination of fluids physical properties. Castille et.al studied on the fabrication of a selfactuated resonant-micro-sensor, based on a thick-film piezoelectric cantilever [14]. Conceptual study for torsional oscillators, which were electromagnetically driven and read out, was presented by Heinisch et al. [15]. They studied feasibility of applying the resonant sensor in viscosity and density measurement of fluids. Heinisch et al., investigated the feasibility of using commercially available steel tuning forks for viscosity and mass density determination [16]. A generalized, reduced order model for resonant viscosity and mass density sensors was discussed by Heinisch et al. [17]. Zhao et.al presented a MEMS resonant sensor to measure fluid viscosity and density based on resonance principle [18]. Payam et al. used a vibrating microcantilever for simultaneous 
viscosity and density measurement of small volumes of liquid [19]. Clara et al. introduced advanced viscosity and density sensor based on diamagnetically stabilized levitation [20]. Gonzalez et.al used mechanical resonators for viscosity and density measurements that could be used in oil and gas applications [21]. Automated high-throughput viscosity and density sensor was presented by Bircher et al. [22]. They used nanomechanical resonators to overcome limitations of classical methods as high sample consumption and long measurement time. Mathematical modeling of a piezoelectrically actuated micro-sensor was presented by Ghanbari et .al in which longitudinally vibrating microbeam with a sensing plate at its end was used for measurement of fluids properties [23].

The fluid media in the mentioned works were modeled based on classical theories which could not properly describe the behavior of fluids in micro and nano scales. Physics of microscale fluids can be described by micro-polar theory developed by Eringen [24-25]. When dimensions a fluid decrease to micrometer size, the rotation of molecules should be taken into account. These rotations are independent of fluid vorticity. In micro-polar theory, effect of couple stresses and consequently the effect of micro-rotations is taken into account. It causes the theory to be introduced as a capable theory in modeling micro scale flows behavior [26-27].

In this paper, a new mathematical model is introduced for measurement of micro-scale fluids physical properties. This model is made up of a shaft with a cylinder at its free end immersed in a micro-scale fluid. Micro cylinder acts as a sensing element. Applying AC voltages to the capacitive plates around the micro-shaft and the outer cylinder can actuate the shaft torsionally. By investigating the effect of surrounding fluid on dynamic behavior of system, this model is introduced as a novel MEMS based sensor for measurement of physical properties of fluids in micro and nano scale.

\section{MICROPOLAR THEORY}

In micro-polar theory, the material points of the fluid are considered to be small deformable particles. As shown in Figure. 1, an element $\Delta V$ is enclosed within its surface $\Delta S$ in the un-deformed body. The center of mass of $\Delta V$ has the position vector $\vec{X}$. The element $\Delta V$ contains $N$ discrete micro-material elements $\Delta V(\alpha)(\alpha=1, \ldots . \mathrm{N})$. The position vector of the center of the mass of macro volume and displacement vector of a material point in the $\alpha$ th microelement in the deformed body are expressed as:

$$
\vec{x}_{k}=\vec{X}_{k}+\vec{U}_{k} ; \vec{U}_{k}^{(\alpha)}=\vec{U}_{k}+\vec{E}_{k}^{(\alpha)} \phi_{k k}
$$

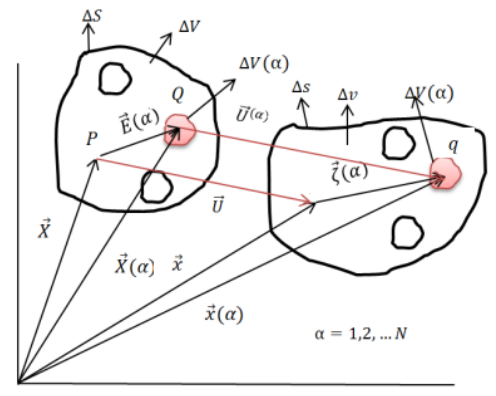

Figure 1. Deformation of a micro-volume in micro-polar theory where $\vec{E}_{k}^{(\alpha)}$ is the position of a point in the microelement relative to the center of mass of $\Delta V$ and $\phi_{k k}$ is defined as skew-symmetric micro-rotation tensor which is independent of macro-rotation tensor, $R_{k k}$. In the micro-polar theory, in addition to macro-strain tensor $E_{K L}$, two micro-strain tensors $\Xi_{K L}$ and $\Gamma_{K M L}$ are defined as [24-25]:

$$
\begin{aligned}
& E_{K L} \equiv \frac{1}{2}\left(\mathrm{C}_{K L}-\delta_{K L}\right)=\frac{1}{2}\left(\mathrm{U}_{L, K}-U_{K, L}\right) \\
& \Xi_{K L} \equiv \psi_{K L}-\delta_{K L}=E_{K L}-\varepsilon_{K L M}\left(R_{M}-\phi_{M}\right) \\
& \Gamma_{K M L} \equiv-\varepsilon_{K L N} \phi_{N, M}
\end{aligned}
$$

where $\mathrm{C}_{K L} \approx U_{L, K}+U_{K, L}+\delta_{K, L}$ is defined as material macro-strain tensor and $\psi_{K L} \approx U_{L, K}+\delta_{K L}+\phi_{K L}, \delta$ is the Kronecker delta, $\varepsilon$ is Levi-Civita symbol and $\Gamma_{K M L} \approx \phi_{K M, L}$ are defined as material micro-strain tensors that are absent in classical theory [24-25].

The equations of the balance of momentum and balance of moment of momentum in micro-polar theory are as following [28]:

$$
\begin{array}{ll}
\sigma_{i j, j}+\rho f_{i}=\rho \ddot{u}_{i} & i=1,2,3 \\
m_{i j, j}+\rho l_{i}-\varepsilon_{i n k} \sigma_{n k}=I_{f} \dot{\phi}_{i} & i=1,2,3
\end{array}
$$

In which $\sigma$ and $m$ are stress and couple stress tensors, $f_{i}$ are body forces per unit mass, $l_{i}$ are body couples per unit mass, $u_{i}$ are displacement components, $\rho$ is mass density, and $I_{f}$ is micro-inertia density, respectively.

The constitutive equations of the micro-polar media link the deformation and micro-rotations tensors to the force and couple stresses as [24-25]:

$$
\begin{aligned}
& \sigma_{k l}=\lambda \delta_{k l} E_{k l}+(2 \mu+k) E_{k l}+k \varepsilon_{k l m}\left(R_{m}-\phi_{m}\right) \\
& m_{k l}=\alpha \delta_{k l} \frac{\partial \phi_{m}}{\partial X_{m}}+\beta \frac{\partial \phi_{k}}{\partial X_{l}}+\gamma \frac{\partial \phi_{l}}{\partial X_{l}}
\end{aligned}
$$

where $\lambda$ and $\mu$ are classical constants. There are 4 extra modulus in micro-polar theory, $\alpha, \beta, \gamma$ and $K$. If these modulus are set equal to zero, the classic continuum media is obtained.

\section{MODEL DESCRIPTION AND ASSUMPTIONS}

As shown in Figure. 2, the proposed model for measurement of micro-scale fluid physical properties is made up of a microshaft and a cylinder as a sensing element at its free end. The sensor can be constructed in both macro and micro scale. The macro scale sensor is used for measurement of density and dynamic viscosity coefficient of the fluid similar to the work that was done by Rezazadeh et.al for classical Newtonian 
fluids where the effect of couple stresses were not taken in to account [7]. The micro scale one is used for measurement of micro-scale fluids vortex and spin gradient viscosity coefficients which are absent in macro-scale fluids. The microshaft and micro-cylinder of the device are assumed to be made of poly crystalline silicone. Capacitive plates are situated around the micro shaft and the outer fixed cylinder. The shaft is actuated via applying $\mathrm{AC}$ voltage to the plates around the shaft and outer fixed cylinder. Two external moments act on the sensing cylinder in measuring process. The first one is the momentum that acts on the sensing cylinder of the microsensor due to the physical properties of the micro-scale fluid and the second one is an external exciting momentum. The sensing cylinder is bounded by the outer fixed cylinder in order to control the magnitude of the shear force. In the proposed mathematical model, it is assumed that the both cylinders have ideally smooth surfaces.
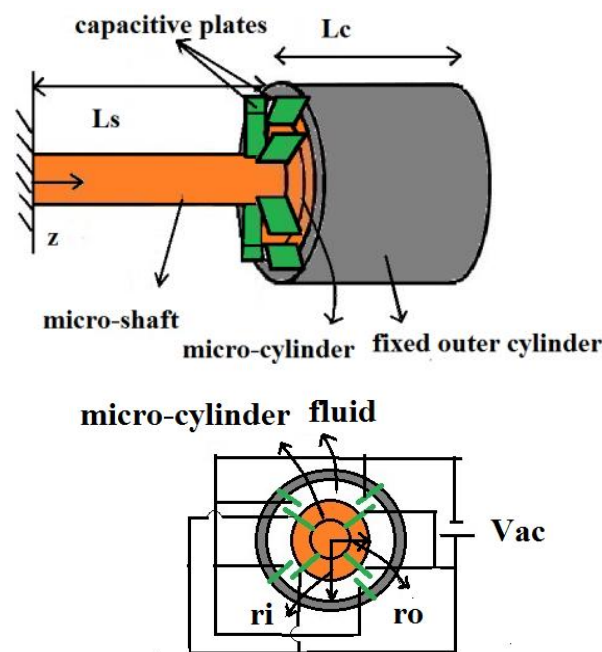

Figure 2. Schematic of the proposed micro-sensor for measurement of micro-scale physical properties

Equation of motion governing angular displacement of the micro-shaft is as following:

$$
\begin{aligned}
& \left.\left(\rho_{s} I_{s}+\tilde{J}_{c} \delta\left(z-L_{s}\right)\right) \frac{\partial^{2} \theta}{\partial t^{2}}-\tilde{G}_{s} I_{s} \frac{\partial^{2} \theta}{\partial z^{2}}=\tilde{T} t\right) \\
& \tilde{T}(t)=-\tilde{\tau}(t) A r_{i} \delta\left(z-L_{s}\right) ; \int_{0}^{L} \tilde{T}(t) \delta\left(z-L_{s}\right) d z=T(t) ; \\
& \int_{0}^{L} \tilde{J}_{c} \delta\left(z-L_{s}\right) d z=J_{c}
\end{aligned}
$$

where, $I_{s}$ is the polar moment of inertia and $\rho_{s}$ is mass density of the shaft. $J_{c}$ is the mass moment of inertia of the cylinder, $L$ is the length of the shaft and $\tilde{\tau}(t)$ is the shear force of the surrounding fluid acting on the surface of the cylinder. $A$ is the surface area of the cylinder, $r_{i}$ is the radius of the cylinder and $T$ is the momentum acting on the surface of the cylinder due to shear force of the surrounding fluid. It should be noted that for simplicity the inertial force owing to the mass of the cylinder and the shear force due to physical properties of the surrounding fluid are assumed to be singular distributed loads with zero load intensity through the whole length of the beam and infinite intensity at its end.

Boundary conditions of equation (3.1) are as:

$\theta(0, t)=0$

$T(L, t)=\left.0 \rightarrow \frac{\partial \theta}{\partial z}\right|_{(L, t)}=\frac{\tilde{T}_{e x}(t)}{I_{s} G_{s}}=T_{e x}(t)$

where $\tilde{T}_{e x}(t)$ is an external exciting momentum acting on the shaft.

Governing equations of the fluid field in the vector form based on micro-polar theory are as following [21]:

$\frac{\partial \rho}{\partial t}+\vec{\nabla} \cdot(\rho \vec{V})=0$

$\left(\lambda+2 \mu+k_{f}\right) \vec{\nabla}(\vec{\nabla} \cdot \vec{V})-\left(\mu+k_{f}\right) \vec{\nabla} \times(\vec{\nabla} \times \vec{V})$

$+k_{f} \vec{\nabla} \times \vec{G}-\vec{\nabla} P+\rho \vec{f}=\rho \overrightarrow{\dot{V}}$

$(\alpha+\beta+\gamma) \vec{\nabla}(\vec{\nabla} \cdot \vec{G})-\gamma \vec{\nabla} \times(\vec{\nabla} \times \vec{G})+k_{f} \vec{\nabla} \times \vec{V}$

$-2 k_{f} \vec{G}+\rho \vec{l}_{f}=\rho I_{f} \overrightarrow{\dot{G}}$

Eq. (3.5) represents conservation of mass; Eq. (3.6) represents conservation of linear momentum; and Eq. (3.7) represents conservation of angular momentum. In the equations above $V$ and $G$ are the fluid velocity and microrotation vectors, $f$ and $l$ are body forces and body couples. $\rho$ is density and $I$ is micro-inertia density of the fluid, $\mu$ and $k_{f}$ are dynamic and vortex viscosity coefficients. $\alpha, \beta, \gamma$ are spin gradient viscosity coefficients and $\lambda$ is second order viscosity coefficient which produces a viscous effect associated with volume change [25-28]. Two important micropolar parameters are length scale of the fluid $\left(\mathrm{L}_{f}\right)$ and coupling parameter $(N)$ which are defined as: $\mathrm{L}_{f}=\sqrt{\frac{\gamma}{4 \mu+2 k_{f}}} \quad$ and coupling parameter $N=\sqrt{\frac{k_{f}}{2 \mu+k_{f}}} ; 0 \leq N \leq 1$. Coupling parameter characterizes coupling between the vortex viscosity coefficient $k_{f}$ and the shear viscosity coefficient $\mu$. If the value of the parameter $N \rightarrow 0$, then the equations of the linear and angular momentums become independent of each other and the linear momentum transforms into the classical Navier-Stocks equations for Newtonian fluids.

By considering the following assumptions:

The fluid is assumed to be incompressible.

There are no body forces and body couples acting along $\theta$ direction.

$\mathrm{r}$ dimension is very small in comparison to $\theta$ and $\mathrm{z}$ directions, so all derivatives with respect to $\theta$ and $\mathrm{z}$ are negligible compared to $\mathrm{r}$ dimension. 
By supposing $I_{s} G_{s}<<I_{c} G_{c}$ in which $I_{c} G_{c}$ is torsional stiffness of the sensing cylinder, the fluid field can be considered one-dimensional.

There is no Pressure gradient in $\theta$ direction.

The Equations (3.6) and (3.7) in the cylindrical coordinate can be rewritten as:

$$
\begin{aligned}
& \rho_{f}\left(\frac{\partial v_{\theta}}{\partial t}\right)=\left(\mu+k_{f}\right)\left[\frac{\partial}{\partial r}\left(\frac{1}{r} \frac{\partial}{\partial r}\left(r v_{\theta}\right)\right)\right]+k_{f}\left[-\frac{\partial g_{z}}{\partial r}\right] \\
& =\left(\mu+k_{f}\right)\left[\frac{\partial^{2} v_{\theta}}{\partial r^{2}}+\frac{1}{r} \frac{\partial v_{\theta}}{\partial r}-\frac{v_{\theta}}{r^{2}}\right]-k_{f} \frac{\partial g_{z}}{\partial r} \\
& \rho_{f}\left(\frac{\partial g_{z}}{\partial t}\right)=-2 k_{f} g_{z}+\gamma\left[\frac{1}{r} \frac{\partial}{\partial r}\left(r \frac{\partial g_{z}}{\partial r}\right)\right]+k_{f}\left(\frac{\partial v_{\theta}}{\partial r}\right) \\
& =-2 k_{f} g_{z}+\gamma\left[\frac{1}{r} \frac{\partial g_{z}}{\partial r}+\frac{\partial^{2} g_{z}}{\partial r^{2}}\right]+k_{f} \frac{\partial v_{\theta}}{\partial r}
\end{aligned}
$$

where $v_{\theta}$ and $g_{z}$ are the fluid velocity and micro-rotation components in $\theta$ and $\mathrm{z}$ directions, respectively.

Boundary conditions of Equations (3.8) and (3.9) are:

$v_{\theta}\left(r_{i}, t\right)=\left.r_{i} \frac{\partial \theta}{\partial t}\right|_{(L, t)}=\alpha(t) ; v_{\theta}\left(r_{o}, t\right)=0$

$g_{z}\left(r_{i}, t\right)=0 ; g_{z}\left(r_{o}, t\right)=0$

Shear force of the fluid acting on the cylinder due to the physical properties of the fluid is:

$\tau\left(r_{i}, t\right)=\left(\mu+k_{f}\right)\left[\frac{\partial v_{\theta}}{\partial r}+\frac{v_{\theta}}{r}\right]_{\left(r_{i}, t\right)}+\left.k_{f} g_{z}\right|_{\left(r_{i}, t\right)}$

By introducing new functions:

$\theta(z, t)=\vartheta(z, t)+z T_{e x}(t)$

$v_{\theta}(r, t)=w_{\theta}(r, t)+\frac{\alpha(t)}{h}\left(r_{o}-r\right) ; h=r_{o}-r_{i}$

Equations. (3.1), (3.8) and (3.9) can be rewritten as: $\lambda(\vartheta(z, t))=\left(\rho_{s} I_{s}+\tilde{J}_{c} \delta\left(z-L_{s}\right)\right)\left(\frac{\partial^{2} \vartheta}{\partial t^{2}}+z \frac{\partial^{2} T_{p}}{\partial t^{2}}\right)$

$-\left(G_{s} I_{s}\right) \frac{\partial^{2} \vartheta}{\partial z^{2}}+$

$\left.\left[\begin{array}{l}\left(\mu+k_{f}\right) \\ -2 \frac{\partial(t)}{h}+\frac{\alpha(t) r_{o}}{r_{i} h}\end{array}\right)\right] A r_{i} \delta\left(z-L_{s}\right)$

$$
\begin{aligned}
& \zeta\left(w_{z}(r, t)\right)=\rho_{f} \frac{\partial \mathrm{w}_{\theta}}{\partial t}+\rho_{f} \frac{\dot{\alpha}(t)}{h}\left(r_{o}-r\right) \\
& -\left(\mu+k_{f}\right)\left(\begin{array}{l}
\frac{\partial^{2} \mathrm{w}_{\theta}}{\partial r^{2}}-\frac{\mathrm{w}_{\theta}(r, t)}{r^{2}} \\
\left.+\frac{1}{r} \frac{\mathrm{w}_{\theta}(r, t)}{\partial r}+\left(-\frac{r_{o}}{h r^{2}}\right) \alpha(t)\right)-k_{f} \frac{\partial g_{z}}{\partial r}
\end{array}\right. \\
& \xi\left(g_{z}(r, t)=\rho_{f}\left(\frac{\partial g_{z}}{\partial t}\right)+2 k_{f} g_{z}-\gamma\left[\frac{1}{r} \frac{\partial g_{z}}{\partial r}+\frac{\partial^{2} g_{z}}{\partial r^{2}}\right]\right. \\
& -k_{f}\left[\frac{\partial \mathrm{w}_{\theta}}{\partial r}-\frac{\alpha(t)}{h}\right]
\end{aligned}
$$

With homogenous Boundary conditions:

$$
\begin{aligned}
& \vartheta(0, t)=0 ;\left.\frac{\partial \vartheta}{\partial z}\right|_{(L, t)}=0 \\
& w_{\theta}\left(r_{o}, t\right)=0 ; w_{\theta}\left(r_{i}, t\right)=0 \\
& g_{z}\left(r_{i}, t\right)=0 ; g_{z}\left(r_{o}, t\right)=0
\end{aligned}
$$

\section{NUMERICAL SOLUTIONS}

In this work, by applying Galerkin reduced order method, approximate solutions are considered as following to solve the coupled equations (3.15), (3.16) and (3.17), respectively

$$
\begin{aligned}
& \vartheta(z, t)=\vartheta_{p}(z, t)=\sum_{k=1}^{p} q_{k}(t) \psi_{k}(z) \\
& \mathrm{w}_{\theta}(r, t)=\left(\mathrm{w}_{\theta}\right)_{m}(r, t)=\sum_{i=1}^{m} a_{i}(t) \phi_{i}(r) \\
& \mathrm{g}_{z}(r, t)=\left(\mathrm{g}_{z}\right)_{n}(r, t)=\sum_{j=1}^{n} b_{j}(t) \varphi_{j}(r)
\end{aligned}
$$

Substituting equations (4.1), (4.2) and (4.3) into equations (3.15), (3.16) and (3.17) yields:

$$
\begin{aligned}
& \lambda(\theta(z, t))=\left((\rho I)_{e q}+\tilde{J}_{c} \delta\left(z-L_{s}\right)\right)\left[\begin{array}{l}
\sum_{k=1}^{p} \ddot{q}_{k}(t) \psi_{k}(z) \\
+z \ddot{T}_{e x}(t)
\end{array}\right]+ \\
& A r_{i} \delta\left(z-L_{s}\right)\left(\mu+k_{f}\right)\left[\begin{array}{l}
\sum_{j=1}^{M} a_{j}(t) \phi_{j}^{\prime}\left(r_{i}\right)+ \\
\left(-\frac{2}{h}+\frac{r_{o}}{r_{i} h}\right)\left(\begin{array}{l}
\sum_{k=1}^{p} \dot{q}_{k}(t) \psi_{k}\left(L_{S}\right) \\
+L_{s} \dot{T}_{e x}(\mathrm{t})
\end{array}\right)
\end{array}\right] \\
& +A \delta\left(z-L_{s}\right) k_{f} \sum_{j=1}^{M} a_{j}(t) \phi_{j}\left(r_{i}\right)=\varepsilon_{1}
\end{aligned}
$$




$$
\begin{aligned}
& \zeta\left(\mathrm{w}_{\theta}(r, t)\right)=\rho_{f} \sum_{j=1}^{M} \dot{a}_{j}(t) \phi_{j}(r)+\frac{\rho_{f}\left(r_{o}-r\right)}{h} \\
& \left(\sum_{k=1}^{p} \ddot{q}_{k}(t) \psi_{k}\left(L_{S}\right)+L_{s} \ddot{T}(\mathrm{t})\right) \\
& -\left(\mu+k_{f}\right)\left(\begin{array}{l}
\sum_{j=1}^{M} a_{j}(t) \phi_{j}^{\prime \prime}(r)+\frac{1}{r^{2}} \sum_{j=1}^{M} a_{j}(t) \phi_{j}(r)+ \\
\left(-\frac{r_{o}}{h r^{2}}\right)\left(\sum_{k=1}^{p} \dot{q}_{k}(t) \psi_{k}\left(L_{S}\right)+L_{s} \dot{T}_{e x}(\mathrm{t})\right) \\
-k_{f} \sum_{j=1}^{p} b_{j}(t) \varphi_{j}^{\prime}(r)=\varepsilon_{2} \\
\xi\left(\omega_{z}(r, t)\right)=\rho_{f} J_{f} \sum_{j=1}^{p} \dot{b}_{j}(t) \varphi_{j}(r) \\
-\gamma\left(\sum_{j=1}^{p} b_{j}(t) \varphi_{j}^{\prime \prime}(r)+\frac{1}{r} \sum_{j=1}^{p} b_{j}(t) \varphi_{j}^{\prime}(r)\right) \\
-k_{f}\left(\sum_{j=1}^{M} a_{j}(t) \phi_{j}^{\prime}(r)-\frac{1}{h}\left(\sum_{k=1}^{p} \dot{q}_{k}(t) \psi_{k}\left(L_{S}\right)\right)\right)=\varepsilon_{3} \\
+L_{s} \dot{T}_{e x}(\mathrm{t})
\end{array}\right)
\end{aligned}
$$

By applying Galerkin- based reduced order model, following ordinary differential equations are obtained:

$\sum_{k=1}^{p} \boldsymbol{M}_{f k}^{(1)} \ddot{q}_{k}+\sum_{k=1}^{p} C_{f k}^{(1)} \dot{q}_{k}+\sum_{k=1}^{p} K_{f k} q_{k} \sum_{i=1}^{m} F_{f i}^{(1)} a_{i}=P_{f}$

$f=1, \ldots . p$

$\sum_{k=1}^{p} M_{s k}^{(2)} \ddot{q}_{k}+\sum_{k=1}^{p} C_{s k}^{(2)} \dot{q}_{k}+\sum_{i=1}^{m} E_{s i}^{(1)} \dot{a}_{i}+\sum_{i=1}^{m} F_{s i}^{(2)} a_{i}$

$+\sum_{j=1}^{n} H_{s j}^{(1)} b_{j}=P_{s} ; s=1, \ldots . m$

$\sum_{k=1}^{p} C_{u k}^{(3)} \dot{q}_{k}+\sum_{i=1}^{m} F_{u i}^{(3)} a_{i}+\sum_{j=1}^{n} G_{u j}^{(1)} \dot{b}_{j}+\sum_{j=1}^{n} H_{u j}^{(2)} b_{j}=P_{u}$

$u=1, \ldots . n$

With the following coefficients:

$$
\begin{aligned}
& M_{f k}^{(1)}=J_{c} \psi_{f}\left(L_{s}\right) \psi_{k}\left(L_{s}\right)+(\rho I)_{e q} \int_{0}^{L_{s}} \psi_{f}(z) \psi_{k}(z) d z \\
& C_{f k}^{(1)}=\left(2 \pi r_{i}^{2}\right)(\mu+k)\left(-\frac{2}{h}+\frac{r_{o}}{r_{i} h}\right) \psi_{f}\left(L_{s}\right) \psi_{k}\left(L_{s}\right) \\
& K_{f k}=-(G I)_{e q} \int_{0}^{L_{s}} \psi_{f}(z) \psi_{k}^{\prime \prime}(z) d z \\
& F_{f i}^{(1)}=\left(2 \pi r_{i}^{2}\right) \psi_{f}\left(L_{s}\right)\left((\mu+k) \phi_{i}^{\prime}\left(r_{i}\right)+k \phi_{i}\left(r_{i}\right)\right) \\
& P_{f}=-\left(J_{c} L_{s} \psi_{f}\left(L_{s}\right)+(\rho I)_{e q} \int_{0}^{L_{s}} z \psi_{f}(z) d z\right) \ddot{T}(t) \\
& -\left(2 \pi r_{i}^{2}\right) L_{s} \psi_{f}\left(L_{s}\right)(\mu+k)\left(-\frac{2}{h}+\frac{r_{o}}{r_{i} h}\right) \dot{T}(t)
\end{aligned}
$$

$$
\begin{aligned}
& M_{s k}^{(2)}=\frac{\rho_{f}\left(r_{o}-r\right)}{h} \psi_{k}\left(L_{s}\right) \int_{r_{i}}^{r_{0}} \phi_{s}(r) d r \\
& E_{s i}^{(1)}=\rho_{f} \int_{r_{i}}^{r_{0}} \phi_{s}(r) \phi_{i}(r) d r \\
& F_{s i}^{(2)}=-(\mu+k)\left(\begin{array}{l}
\int_{r_{i}}^{r_{0}} \phi_{s}(r) \phi_{i}^{\prime \prime}(r) d r+ \\
\int_{r_{i}}^{r_{0}} \frac{1}{r^{2}} \phi_{s}(r) \phi_{i}(r) d r
\end{array}\right) \\
& H_{s j}^{(1)}=-k \int_{r_{i}}^{r_{o}} \phi_{s}(r) \varphi_{j}^{\prime}(r) d r \\
& P_{s}=-\left(\frac{\rho_{f}\left(r_{o}-r\right)}{h} L_{s} \int_{r_{i}}^{r_{o}} \phi_{s}(r) d r\right) \ddot{T}(t) \\
& +\frac{(\mu+k)}{h} L_{s}\left(\int_{r_{i}}^{r_{0}} \frac{1}{r} \phi_{s}(r) d r-\int_{r_{i}}^{r_{0}} \frac{r_{o}}{r^{2}} \phi_{s}(r) d r\right) \dot{T}(t) \\
& C_{u k}^{(3)}=\frac{k}{h} \psi_{k}\left(L_{s}\right) \int_{r_{i}}^{r_{o}} \varphi_{u}(r) d r \\
& F_{u i}^{(3)}=-k \int_{r_{i}}^{r_{o}} \varphi_{u}(r) \varphi_{i}^{\prime}(r) d r \\
& G_{u j}^{(1)}=\rho_{f} I_{f} \int_{r_{i}}^{r_{o}} \varphi_{u}(r) \varphi_{j}(r) d r \\
& H_{u j}^{(2)}=-\gamma\left(\int_{r_{i}}^{r_{o}} \varphi_{u}(r) \varphi_{j}^{\prime \prime}(r) d r+\int_{r_{i}}^{r_{o}} \frac{1}{r} \varphi_{u}(r) \varphi_{j}^{\prime}(r) d r\right) \\
& P_{u}=\left(-\frac{k}{h} L_{s} \int_{r_{i}}^{r_{o}} \varphi_{u}(r) d r\right) \dot{T}(t)
\end{aligned}
$$

\section{NUMERICAL RESULTS}

In applying Galerkin method for solving the coupled equations of micro-shaft vibration and fluid flow, Shape functions are considered as following which satisfy the boundary conditions (3.18), (3.19) and (3.20), respectively. So:

$$
\begin{aligned}
& \psi_{k}(z)=\sin \left(\frac{(2 k-1) \pi}{2 L} z\right) ; \phi_{i}(r)=\sin \left(\frac{i \pi}{h}\left(r-r_{i}\right)\right) \\
& \varphi_{j}(r)=\sin \left(\frac{j \pi}{h}\left(r-r_{i}\right)\right)
\end{aligned}
$$

Geometric and material properties of the proposed model in this study are listed in Table1.

Table 1. Geometrical and material properties of the proposed model

\begin{tabular}{ccc}
\hline Properties & Micro-shaft & Cylinder \\
\hline Length & $100 \mu \mathrm{m}$ & $100 \mu \mathrm{m}$ \\
\hline Diameter & $20 \mu \mathrm{m}$ & $50 \mu \mathrm{m}$ \\
\hline Young's modulus & $169 \mathrm{GPa}$ & $169 \mathrm{GPa}$ \\
\hline Poisson's modulus & 0.27 & 0.27 \\
\hline Mass density & $2331 \mathrm{~kg} \cdot \mathrm{m}^{-3}$ & $2331 \mathrm{~kg} . \mathrm{m}^{-3}$ \\
\hline
\end{tabular}

Table 2 shows the first natural frequency of the micro-shaft in the absence of the fluid shear force, for different number of 
the used shape functions. The fluid gap $(h)$ is considered to be $20 \mu \mathrm{m}$. Calculation presents that for $\mathrm{p}=6$ the results converge and show $2.2 \%$ error in comparison with the first natural frequency of the shaft having a concentrated mass at the free end.

Table 2. Values of the first calculated natural frequency of the system (MHZ)

\begin{tabular}{cc}
\hline $\begin{array}{c}\text { Number of shape } \\
\text { functions }\end{array}$ & $\begin{array}{c}\text { Natural } \\
\text { Frequency }\end{array}$ \\
\hline$p=1$ & 1.5 \\
\hline$p=2$ & 1.43 \\
\hline$p=3$ & 1.41 \\
\hline$p=4$ & 1.4 \\
\hline$p=5$ & 1.39 \\
\hline$p=6$ & 1.39 \\
\hline
\end{tabular}

Figure 3 presents the effects of micro-scale fluid coupling parameter on torsional vibration of the micro-shaft. Results show that in fluids with high values of vortex viscosity coefficient, the micro-rotations have more dependency to macro-rotations that causes damping and inertial effects of fluid to increase. Consequently, Resonance frequency and resonance amplitude changes of the micro-shaft are more considerable in fluids with high vortex viscosity coefficient.

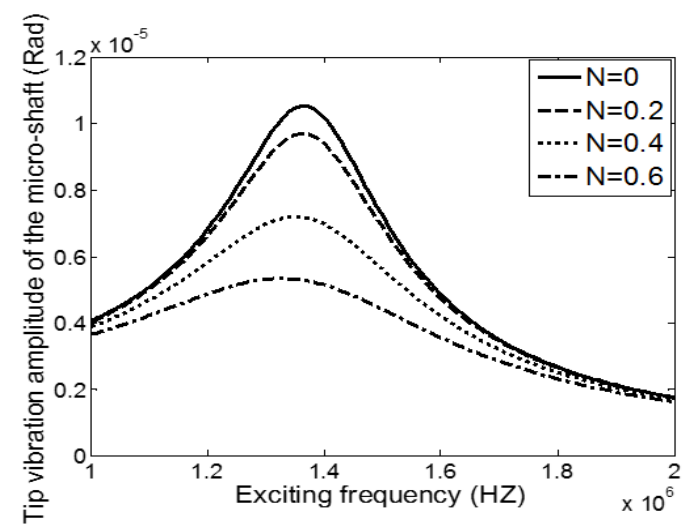

Figure 3. Tip vibration amplitude of the micro-beam versus exciting frequency for different values of coupling parameter

Considering geometrical and material parameters of sensor listed in table.1, knowing kinematic viscosity and density of the fluid via classical methods, the effect of length scale of fluid on frequency response of the micro-shaft for different values of spin gradient viscosity coefficients are investigated, and results are shown in Figure 4 and Figure 5. As mentioned above fluid loading changes the resonance frequency and resonance amplitude of the shaft. so, for the length scale in the range of approximate $L_{f} \leq 1.4 e-6$, the changes of microshaft resonance frequency are considerable. Therefore, in the mentioned ranges of fluid length scale, this sensor can be applied to measure micro-scale fluid vortex and spin gradient viscosity coefficients simultaneously. For fluids having higher values of length scales, the vibrating microshaft doesn't have a considerable resonance frequency changes, therefore it is difficult to measure vortex and spin gradient viscosity coefficients of fluids with hight length scale simultaneously. However, by knowing the vortex viscosity coefficient of a fluid, it's spin viscosity coefficient can be determined, and it is also valid on the contrary. It should be noted that the obtained results are dependent of the sensor geometry and also the amplitude of the exciting momentum. For obtaining uncertainty of measuring, we can say that capacitive plates around the vibrating micro shaft and the fixed micro cylinder form a capacitor. Variation of the capacitance of the capacitor caused by torsional vibration of the microshaft can show the uncertainty of measurement. As todays variation of capacitance of the capacitor can be measured up to 40e-16 farad with high resolution, so measurement of desired values from measurement characteristics can be done with high accuracy [29].

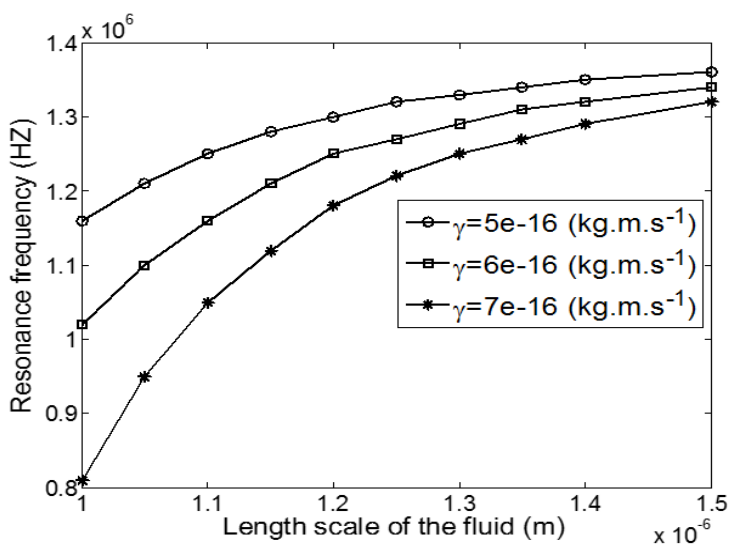

Figure 4. Resonance frequency versus length scale considering micro-scale-fluid with different spin gradient viscosity coefficient

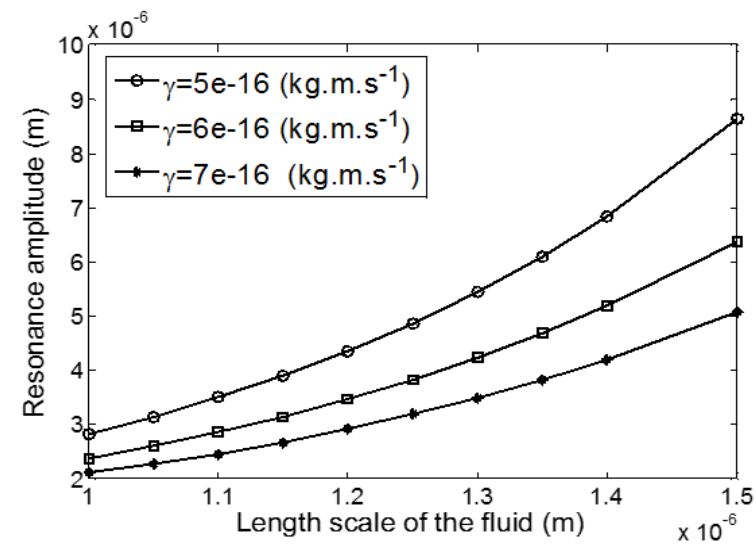

Figure 5. Resonance amplitude versus length scale considering micro-scale-fluids with different spin gradient viscosity coefficients

\subsection{Effect of fluid gap}

For this study, resonance frequency and resonance amplitude changes of the micro-sensor in first mode of vibration for different values of fluid gap are determined and shown in Figure 6 and Figure 7. We can see that decreasing fluid gap, due to increasing inertial and damping effect of fluid, decreases resonance frequency and amplitude of the microsensor. Results also show that in lower values of fluid gap, resonance frequency and resonance amplitude changes are more considerable than in higher values. Therefore, in a sensor with small gap size due to having higher sensibility, 
measurement of fluid properties can be obtained with high accuracy than in a sensor with large gap size.

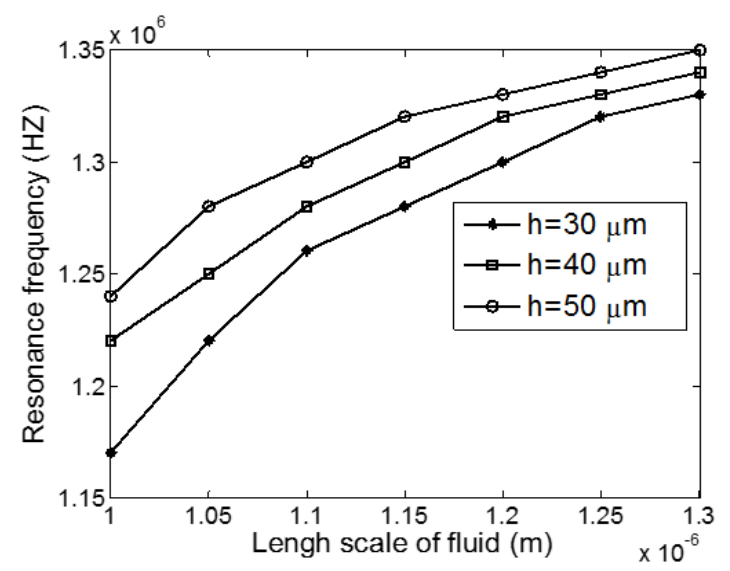

Figure 6. Resonance frequency versus length scale for different values of fluid gap

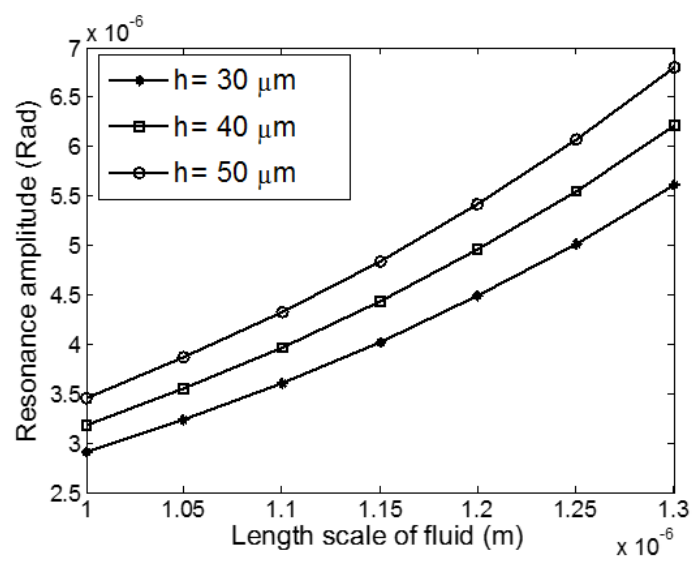

Figure 7. Resonance amplitude versus length scale for different values of fluid gap

For this study, resonance frequency and resonance amplitude changes of the micro-sensor for different values of sensing cylinder surface area are determined and the obtained results are shown in Figure 8 and Figure 9.

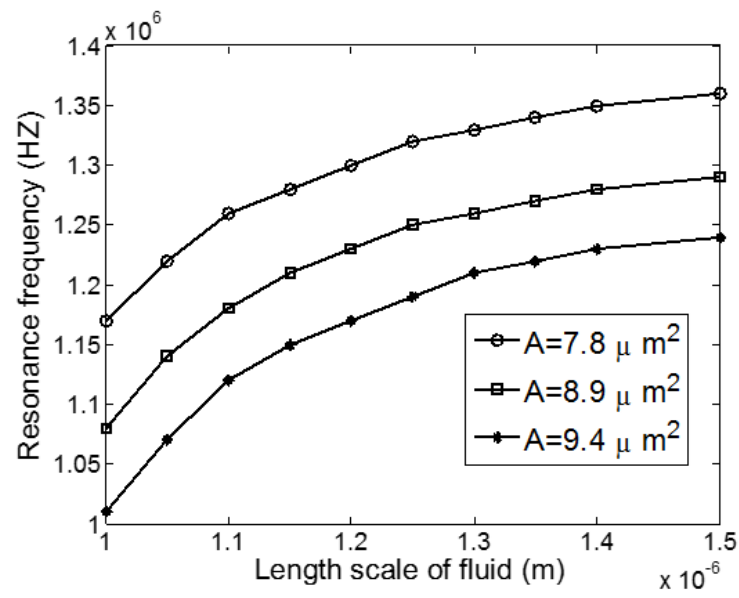

Figure 8. Resonance frequency versus length scale for different values of sensing cylinder area

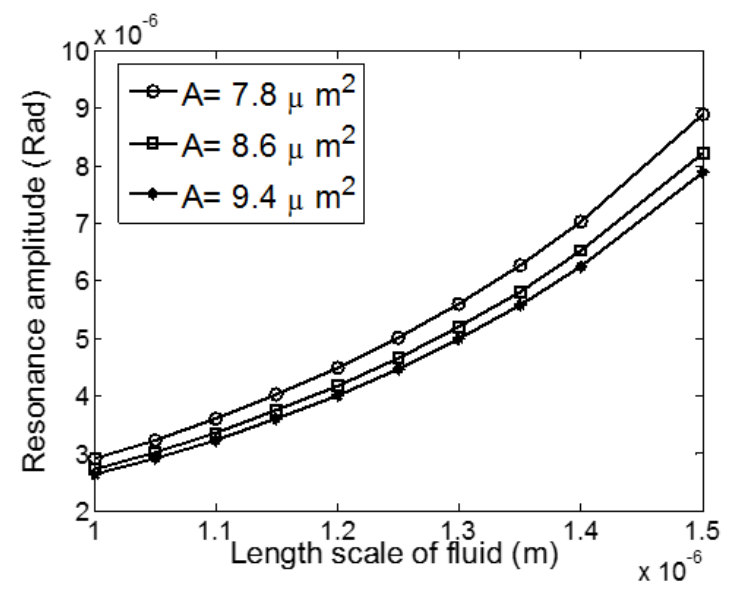

Figure 9. Resonance amplitude versus length scale for different values of sensing cylinder area

Results show that in a sensor with large sensing element area, changes of the resonance frequency are more sensible in comparison to the sensor having a small sensing element area. It means that a sensing element with larger surface area causes inertial and damping effects of the fluid and sensibility of the micro-sensor to increase. Therefore, replacing the sensing element with a one having large surface area allows measuring the viscosity coefficients of a micro-scale fluid with small uncertainty.

\section{CONCLUSION}

In this paper, a novel MEMS-based sensor was presented for measurement of micro-scale fluid physical properties. A mathematical model was introduced which could be actuated torsionally via applying an AC voltage to the capacitive plates around the shaft and the outer fixed cylinder. After deriving governing equations of motion of the shaft and the fluid media and transforming them to an enhanced form, they were discretized applying Gelerkin-based reduce order model. Solving coupled differential equations simultaneously showed that a micro-scale fluid has dissipative and inertial effects on torsional vibration of the micro-shaft. This causes resonance frequency and resonance amplitude of the shaft to decrease, therefore by knowing kinematic viscosity of a fluid, two other viscosity coefficients of a micro-scale fluid can be measured by detecting changes in resonance frequency and resonance amplitude of the micro-shaft. The effects of the fluid gap and sensing cylinder surface area on the dynamic response of the micro-shaft were investigated. Results showed that a sensor having small fluid gap and large surface area has considerable resonance frequency and amplitude changes and consequently has higher sensibility. The sensing element surface area, the fluid gap, and the amplitude of the exciting momentum acting on the shaft affect the sensibility. Replacing the sensor with one having large surface area and small fluid gap can measure the viscosity coefficients with desired accuracy.

\section{REFERENCES}

[1] Ebadian MA, Dillon J, Moore J, Jones K. (1996). Sensors for viscosity and shear strength measurement. Technical 
Report. Florida International Univ., Miami, FL (United States). https://doi.org/10.2172/666055

[2] Stoyanov PG, Grimes CA. (2000). A remote grey magnetostrictive viscosity sensor. Sensor and Actuators A 80(1): 8-14.

[3] Martin BA, Wenzel SW, Richard MW. (1990). Viscosity and density sensing with ultrasonic plate waves. Sensor and Actuators A 22: 704-708 https://doi.org/10.1016/S0924-4247(99)00288-5

[4] Rezazadeh G, Khatami F, Tahmasebi A. (2007). Investigation of thetorsion and bending effects on static stability of electrostatictorsional micromirrors. Microsystem Technologies 13(7): 715-722. https://doi.org/10.1007/s00542-006-0362-1

[5] Saif MTA, Alaca BE, Sehitoglu H. (1999). Analytical modeling of electrostatic membrane actuator micro pumps. Journal of Microelectromechanical Systems 8(3): 335-345. https://doi.org/10.1109/84.788638

[6] Bao M, Wang W. (1996). Future of Microelectro mechanical systems (MEMS). Sensors and Actuators A 56(1): 135-141. https://doi.org/info:doi/10.1016/09244247(96)01274-5

[7] Rezazadeh G, Ghanbari M, Mirzaee I. (2010). On the modeling of piezoelectrically actuated microsensor for Simultaneous measurement of fluids viscosity and density. Measurement 43(10): 1516-1524. https://doi.org/10.1016/j.measurement.2010.08.022

[8] Ghanbari M, Hossainpour S, Rezazadeh G. (2015). Study of squeeze film damping in a microbeam resonator based on micropolar theory. Latin American Journal of Solids and Structures 12(1): 77-91.

[9] Ghanbari M, Hossainpour S, Rezazadeh G. (2015). Studing thin film damping in a micro-beam resonator based on non-classical theories. Acta Mechanica Sinica 32(3): 369-379. https://doi.org/10.1007/s10409-0150482-x

[10] Rezazadeh G, Ghanbari M. (2018). On the mathematical modeling of a MEMS-based sensor for simultaneous measurement of fluids viscosity and density. Sensing and Imaging 19: 27. https://doi.org/10.1007/s11220-0180213-z

[11] Enoksson P, Stemme G, Stemme E. (1995). Fluid density sensors based on resonance vibration. Sensors and Actuators A 47(1-3): 327-331. https://doi.org/10.1016/0924-4247(94)00915-5

[12] Sader JE. (1998). Frequency response of cantilever beams immersed in viscous fluids with applications to the atomic force microscopy. Journal of Applied Physics 84(64): 64-76. https://doi.org/10.1063/1.368002

[13] Agoston A, Keplinger F, Jakopy B. (2005). Evaluation of a vibrating micromachined cantilever sensor for measuring $g$ the viscosity of complex organic liquids. Sensors and Actuators A 123: 82-86. https://doi.org/10.1016/j.sna.2005.02.020

[14] Castille CH, Dufour I, Lucat IC. (2010). Longitudinal vibration mode of piezoelectric thick-film cantileverbased sensors in liquid media. Applied Physics Letters 96: 154102. https://doi.org/10.1063/1.3387753

[15] Heinisch M, Voglhuber-Brunnmaier T, Reichel EK, Dufour I, Jakoby B. (2015). Electromagnetically driven torsional resonators for viscosiry and mass density sensing applications. Sensora and Actuators A 229(15): 182-191. https://doi.org/10.1016/j.sna.2015.03.033
[16] Heinisch M, Voglhuber-Brunnmaier T, Reichel, EK, Dufour I, Jakobe B. (2015). Application of resonant tuning forks with circular and rectangular crosssections for precise mass density and viscosity measurements. Sensors and Actuators A 226(1): 163-174. https://doi.org/10.1016/j.sna.2015.02.007

[17] Heinisch M, Reichel EK, Dufour I, Jakoby B. (2014). Modeling and experimental investigation of resonant viscosity and mass density sensors considering their cross sensitivity to temperature. Procedia engineering 87: 472-475. https://doi.org/10.1016/j.proeng.2014.11.391

[18] Zhao L, Hu Y, Wang T, Ding J, Liu X, Zhao Y, Jiang Z. (2016). A MEMS resonant sensor to measure fluid density and viscosity under flexural and torsional vibrating modes. Sensors 16(6): 830. https://doi.org/10.3390/s16060830

[19] Payam AF, Trewby W, Voitchovsky K. (2017). Simultaneous viscosity and density measurement of small volumes of liquids using a vibrating microcantilever. Analyst 142(9): 1492-1498. https://doi.org/10.1039/C6AN02674E

[20] Clara S, Antlinger H, Abdallah A, Reichel E, Hilber W, Jakoby B. (2016). An advanced viscosity and density sensor based on diamagnetically stabilized levitation. Sensora and Actuators A 248: 46-53. https://doi.org/10.1016/j.sna.2016.07.021

[21] Gonzalez M, Seren HR, Ham G, Buzi E, Bernero G, Deffenbaugh M. (2018). Viscosity and density measurements using mechanical osillators in oil and gas applications. IEEE Transactions on Instrumentation and Measurement 67(4): 804-807. https://doi.org/10.1109/TIM.2017.2761218

[22] Bircher B, Krenger R, Braun T. (2016). Automated highthroughput viscosity and density sensor using nanomechanical resonators. Sensora and Actuators B 223: 784-790. https://doi.org/10.1016/j.snb.2015.09.084

[23] Ghanbari M, Hossainpour S, Rezazadeh G. (2015). On the modeling of a piezoelectrically actuated micro-sensor for measurement of microscale fluid physical properties. Applied Physics A 121(2): 651-663.

[24] Eringen AC. (1996). Theory of micro-polar fluids. Journal of Mathematics and Mechanics 16(1): 1-18. https://doi.org/10.1512/iumj.1967.16.16001

[25] Erigena AC. (1972). Theory of thermomicrofluids. Journal of Mathematical Analysis and Applications 38(2) 480-496. https://doi.org/10.1016/0022-247X(72)901060

[26] Kucaba-Pietal A. (2008). Applicability of the micropolar fluid theory in solving microfluidics problems. Proceedings of 1st European Conference on Microfluidics, Bologna.

[27] Chen J, Liang C, Lee JD. (2011). Theory and simulation of micropolar fluid dynamics. Journal of Nanoengineering and Nanosystems 224: 31-39. https://doi.org/10.1177/1740349911400132

[28] Ahmadi G. (1976). Self-similar solution of incompressible micro-polar boundary layer flow over a semi-infinite plate. International Journal of Engineering Science 14(7): 639-646. https://doi.org/10.1016/00207225(76)90006-9

[29] Song X, Fang JC, Sheng W. (2009). Circuit for closeloop capacitive micro accelerometers. Journal of Beijing University of Aeronautics and Astronautics 35(3): 384388. 
NOMENCLATURE

C

E

$f$

G

$g$

h

I

$J$

k

L

N

$r$

R

$T$

u

$v$

X

$a, b$ material macro-strain tensor

macro-strain tensor

Body force per unit mass

micro-roration vector

micro-roration component

fluid gap

micro-inertia density

mass moment of inertia

Vortex viscosity coefficient

Body couples per unit mass

Lengh

Lengh scale

Coupling parameter

radius

macro-rotation tensor

momentum

displacement $(\mathrm{m})$

Fluid velocity

Position of element

Functions

\section{Greek symbols}

$\alpha, \beta, \gamma \quad$ Spin gradient viscosity coefficient

$\lambda$

$\mu$

$\rho$

$\phi$

$\Xi$

$\Gamma$

$\psi$

$\sigma$

$m$

$\delta$

$\varepsilon$

$\theta$

$\vartheta, \varphi, \phi, \psi$

$\tau$

second order viscosity coefficient dynamic viscosity coefficient

Density, kg. $\mathrm{m}^{-3}$

micro-rotation tensor

micro-strain sensor

material micro-strain sensor

material micro-strain sensor

Stress tensor

Couple stress tensor

the Kronecker delta

Levi-Civita symbol

Angular displacement, Rad

functions

shear stress

\section{Subscripts}

$\begin{array}{ll}c & \text { cylinder } \\ f & \text { fluid } \\ s & \text { shaft }\end{array}$

